\title{
VIRTUAL SCREENING OF HETEROCYCLIC COMPOUNDS AGAINST ANGIOTENSIN-CONVERTING ENZYME FOR POTENTIAL ANTIHYPERTENSIVE INHIBITORS
}

\author{
ZOZIMUS DIVYA LOBO C ${ }^{1}$, SYED MOHAMED A², GNANENDRA SHANMUGAM ${ }^{3 *}$
}

\begin{abstract}
${ }^{1}$ Department of Chemistry, St. Mary's College (Autonomous), Tuticorin, Tamil Nadu, India, Affiliated to Manonmanium Sundaranar University, Tirunelveli, Tamil Nadu, India. ${ }^{2}$ Department of Chemistry, Sadakathullah Appa College (Autonomous), Tirunelveli, Tamil Nadu, India. ${ }^{3}$ Department of Biotechnology, Mahendra Arts and Science College, Namakkal, Tamil Nadu, India. Email: gnani.science@gmail.com

Received: 30 August 2018, Revised and Accepted: 30 October 2018
\end{abstract}

ABSTRACT

Objective: The objective of this study was to investigate the antihypertensive activity of heterocyclic compounds against angiotensin-converting enzyme (ACE) through molecular docking studies.

Methods: The X-ray crystal three-dimensional (3D) structure of human ACE complexed with lisinopril (PDB ID: 1086) was retrieved from protein databank. The two-dimensional structures of 10 selected heterocyclic compounds were drawn in ACD-Chemsketch and converted into 3D structures. The 3D structures of compounds were virtually screened in the binding pockets of ACE using FlexX docking program. Further, the chemical entities revealing the molecular electronic structures of the best docked compound (Compound-4) were explored through density functional theory studies.

Results: The Compound-4 showed the highest docking score of $-26.6290 \mathrm{~kJ} / \mathrm{mol}$ with ACE. The Hbond and non-bonded interactions are favored by phenylalanine, leucine, and arginine. The energy gap of $1.60 \mathrm{eV}$ between highest occupied molecular orbital and lowest unoccupied molecular orbitals explained the presence of strong electron-acceptor group. Furthermore, the molecular electrostatic potential studies clearly envisaged the requirement of electropositive and electronegative groups are crucial for the ACE inhibitor activities.

Conclusion: The identification of good ACE inhibitors requires the understanding of the current ACE inhibitors. Thus, the docking interactions of Compound-4 and its molecular electronic structure significantly imply its potential as antihypertensive agent. However, further clinical studies are required to ascertain its potential toxic effects.

Keywords: Angiotensin-converting enzyme, Angiotensin-converting enzyme inhibitors, Docking, Density functional theory studies, Highest occupied molecular orbital, Lowest unoccupied molecular orbitals, Molecular electrostatic potentials.

(C) 2019 The Authors. Published by Innovare Academic Sciences Pvt Ltd. This is an open access article under the CC BY license (http://creativecommons. org/licenses/by/4. 0/) DOI: http://dx.doi.org/10.22159/ajpcr.2019.v12i2.29106

\section{INTRODUCTION}

In recent years, cardiovascular diseases have become a serious problem worldwide. The World Health Organization has reported an increase in the number of patients suffering from this disease. Hypertension is an important risk factor, contributing to other cardiovascular diseases such as blood vessel disorders, coronary heart disease, aortic aneurysm, and stroke [1]. Cardiac arrhythmia is another cardiovascular problem. An arrhythmia is any disorder of the heart rate that may cause stroke. Heart rhythm's disorders may be caused by genetic factors or occur when the heart muscle (myocardium) is damaged, sometimes by hypertension [2].

At present, existing treatments for high blood pressure (BP) are not very effective and are generally uncomfortable for patients. This relies in that the patient needs to have a very strict control in the dosage and in the moment of the administration of the drug [3]. Moreover, also, some patients have an unfavorable response after the administration, leading them to a fast BP reduced. One of the most widely used compounds for the treatment of hypertension is Captopril. Like many others on the market, this drug was designed with computational tools. Over the past few decades, computational studies, together with rational drug design, have become a critical part in the development of new drugs.

Raised BP, especially systolic pressure (hypertension), confers a significant cardiovascular risk and public health concern and should be actively treated. One of the major systems involved in the elevation of the pressure is the renin-angiotensin system (RAS), and subsequently, its inhibition will have beneficial effects to lower BP and improve cardiovascular health [4]. The RAS is regulated by a series of highly specific enzymatic reactions. The first enzymatic reaction in the pathway starts with renal production of renin that cleaves angiotensinogen to generate angiotensin I. Angiotensin I is then cleaved by angiotensin-converting enzyme (ACE) to generate the active peptide vasoconstrictive hormone angiotensin II.

Many ACE inhibitors are known to be useful in the treatment of hypertension. The search for ACE inhibitors that lacked the sulfhydryl group also leads to the investigation of phosphorus-containing compounds. The phosphinic acid is capable of binding to ACE in a manner similar to enalapril. The interaction of the zinc atom with the phosphinic acid is similar that is seen with sulfhydryl groups. The purpose of the study is to determine the ability of some derivatives as ACE inhibitors through docking studies and the derivative with the highest potency and understanding its chemical entities through density functional theory (DFT) studies might pave path to design of novel ACE inhibitors with potential inhibition activity.

\section{METHODS}

Ligand selection

A total of 10 antihypertension inhibitor molecules reported in the studies of Yu et al., 2015, were selected in this study to explore their chemical entities required for the ACE inhibitory activity. The twodimensional (2D) structures of compounds drawn in ACD-Chemsketch (Version 12) [5] were obtained as simplified molecular-input line-entry system (SMILES). Further, the three-dimensional (3D) structures were generated and converted into standard delay file format at "online SMILES convertor and Structure file generator" server [6]. 
Target and binding site prediction

The X-ray crystal structure of human ACE complexed with lisinopril (PDB ID: 1086) [7] was retrieved from protein databank [8]. The amino acid residues within 6 Å radius of reference ligand lisinopril was considered as binding site using LeadIT (Version 2.1.9) [9].

\section{Virtual screening}

The 3D structures of all the 10 selected ACE inhibitors were virtually screened to reveal their binding efficiencies through docking in the predicted binding site of ACE using FlexX module of LeadIT. The docking was performed with the default parameters [10-12].

\section{Docking interactions}

The docking interactions revealing Hbond and Van der Waal forces among the selected ACE inhibitors and the amino acid residues of ACE were analyzed using pose-view [13] module of LeadIT.

\section{DFT studies}

The molecular structure of the best docked compound was computed using Gaussian software to retrieve the molecular geometric coordinates. Both DFT and time-dependent DFT calculations were performed using Gaussian 03W software. B3LYP hybrid functional 6-311G (d) basis set was used in the calculation methods. The models of the electron density of various energy levels of the compound were visualized using Gauss View 3.0 [14].

\section{RESULTS AND DISCUSSION}

Hypertension is a highly prevalent cardiovascular risk factor and an increase in BP increases the risk of developing heart disease, obesity, kidney disease, eye damage, and stroke. Excessive salt intake has been suspected as a cause of high prevalence of hypertension. A high dietary salt intake contributes to the risk of hypertension, which further antagonizes the BP-lowering effect of most antihypertensive drugs. It has been reported that a combination of diet rich in fruits, vegetables, and low-fat dairy products could substantially lower BP levels [15].

Vázquez-Valadez et al. [16] reported that main target in the treatment of hypertension is the ACE. This enzyme is responsible for producing angiotensin II, a potent vasoconstrictor. Therefore, one of the targets in the treatment of hypertension is to inhibit ACE activity. Hence, this study's aim is to use computational studies and to demonstrate that the heterocyclic compounds have a molecular affinity for ACE and are capable of inhibiting ACE activity, thus avoiding the production of the vasopressor angiotensin II. The 3D structure of ACE protein as target is shown in Fig. 1 and the 2D structures of 10 selected heterocyclic compounds considered in this study are shown in Fig. 2.

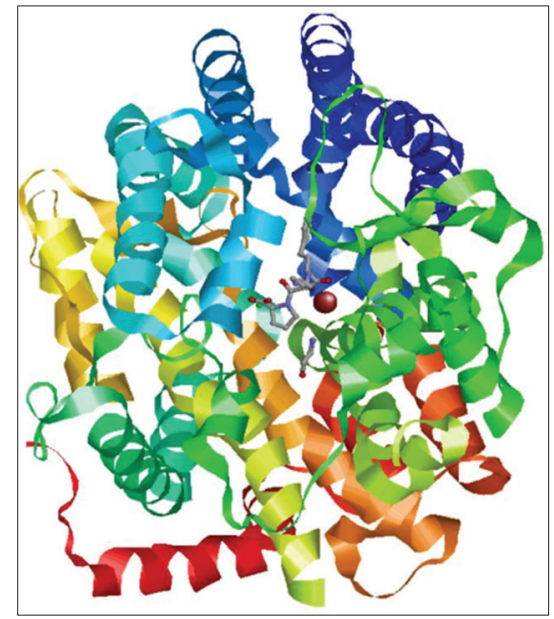

Fig. 1: The protein structure of human angiotensin-converting enzyme complexed with lisinopril (PDB ID: 1086)

\section{Docking interactions}

The docking interactions of all the selected compounds in the study exhibited theoretically encouraging docking scores. Among the selected compounds, Compound- 4 has exhibited relatively good binding affinity as compared to the other ACE inhibitors considered in this study. The docking interactions of the each compound exploring the amino acids favoring Hbond interactions and non-bonded interactions and their docking scores are discussed.

\section{Docking interactions of Compound-1}

The Compound- 1 showed the docking score of $-11.3060 \mathrm{~kJ} / \mathrm{mol}$ with the ACE. It is observed that the Compound- 1 exhibited critical interactions with the catalytic amino acid residues present in the active site cavity of the ACE by favoring the Hbond interactions and non-bonded interactions. The amino acid Leu380, Phe381, and Arg110 favored the Hbond interactions and the non-bonded interactions are supported by Phe130, Phe487, Phe381, Leu382, Glu383, and Arg110. Thus, this compound can be considered as potential ACE inhibitor molecule. The docking complex and docking interactions of Compound-1 within the active site of ACE is shown in Fig. 3a.

\section{Docking interactions of Compound-2}

The Compound-2 showed the docking score of $-17.9469 \mathrm{~kJ} / \mathrm{mol}$ with the ACE. It is observed that the Compound-2 exhibited critical interactions with the catalytic amino acid residues present in the active site cavity of the ACE by favoring the Hbond interactions and non-bonded interactions. The amino acid Phe381, Arg110, and Leu380 favored the Hbond interactions and the non-bonded interactions are supported by Arg110, Glu383, Leu382, Phe130, Phe487, and Phe381. Thus, this compound can be considered as potential ACE inhibitor molecule. The docking complex and docking interactions of Compound-2 within the active site of ACE is shown in Fig. 3b.

\section{Docking interactions of Compound-3}

The Compound-3 showed the docking score of $-18.9100 \mathrm{~kJ} / \mathrm{mol}$ with the ACE. It is observed that the Compound-3 exhibited critical interactions with the catalytic amino acid residues present in the active site cavity of the ACE by favoring the Hbond interactions and non-bonded interactions. The amino acid Phe381, Leu380, and Arg110 favored the Hbond interactions and the non-bonded interactions are supported by Phe487, Arg110, Leu382, Phe381, Glu383, and Phe130. Thus, this compound can be considered as potential ACE inhibitor molecule. The docking complex and docking interactions of Compound-3 within the active site of ACE is shown in Fig. 3c.

\section{Docking interactions of Compound-4}

The Compound -4 showed the highest docking score of $-26.6290 \mathrm{~kJ} \mathrm{~mol}$ with the ACE. It is observed that the Compound-4 exhibited critical interactions with the catalytic amino acid residues present in the active site cavity of the ACE by favoring the Hbond interactions and non-bonded interactions. The amino acid Phe381, Leu380, and Arg110 favored the Hbond interactions and the non-bonded interactions are supported by Arg110, Glu383, Leu382, Phe130, Phe487, and Phe381. Thus, this compound can be considered as potential ACE inhibitor molecule. The docking complex and docking interactions of Compound-4 within the active site of ACE is shown in Fig. 3d.

\section{Docking interactions of Compound-5}

The Compound- 5 showed the docking score of $-14.2283 \mathrm{~kJ} / \mathrm{mol}$ with the ACE. It is observed that the Compound- 5 exhibited critical interactions with the catalytic amino acid residues present in the active site cavity of the ACE by favoring the Hbond interactions and non-bonded interactions. The amino acid Phe381, Leu380, and Arg110 favored the Hbond interactions and the non-bonded interactions are supported by Arg110, Glu383, Leu382, Phe130, Phe487, and Phe381. Thus, this compound can be considered as potential ACE inhibitor molecule. The docking complex and docking interactions of Compound-5 within the active site of ACE is shown in Fig. 3e. 


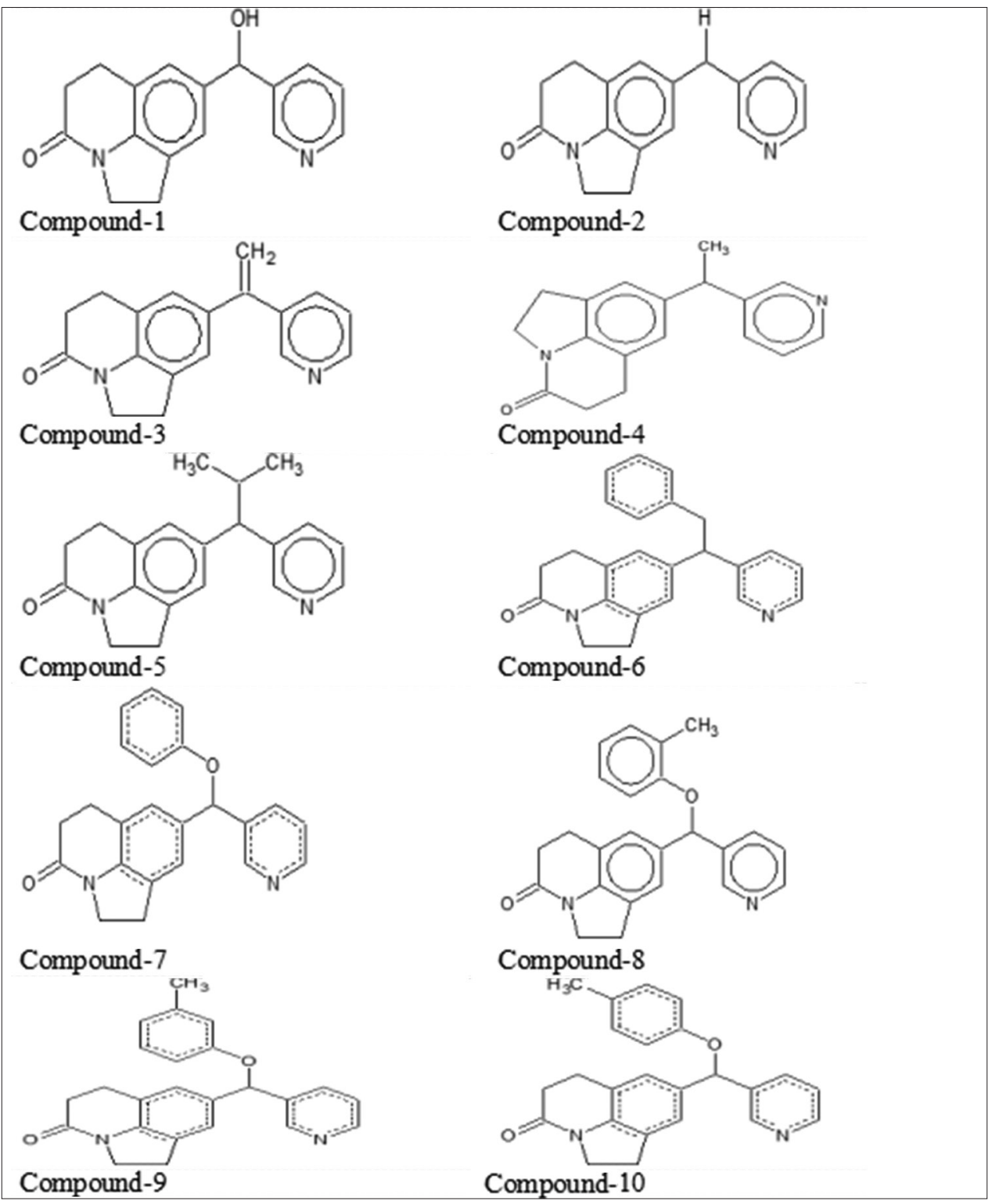

Fig. 2: The two-dimensional structure of 10 selected heterocyclic compounds

\section{Docking interactions of Compound-6}

The Compound- 6 showed the docking score of $-11.1612 \mathrm{~kJ} / \mathrm{mol}$ with the ACE. It is observed that the Compound- 6 exhibited critical interactions with the catalytic amino acid residues present in the active site cavity of the ACE by favoring the Hbond interactions and non-bonded interactions. The amino acid Phe381, Leu380, and Arg110 favored the Hbond interactions and the non-bonded interactions are supported by Arg110, Glu383, Leu382, Phe130, Phe487, and Phe381. Thus, this compound can be considered as potential ACE inhibitor molecule. The docking complex and docking interactions of Compound-6 within the active site of ACE is shown in Fig. $3 f$.

\section{Docking interactions of Compound-7}

The Compound-7 showed the docking score of $-15.9906 \mathrm{~kJ} / \mathrm{mol}$ with the ACE. It is observed that the Compound-7 exhibited critical interactions with the catalytic amino acid residues present in the active site cavity of the ACE by favoring the Hbond interactions and non-bonded interactions. The amino acid Phe381, Leu380, and Arg110 favored the Hbond interactions and the non-bonded interactions are supported by Arg110, Glu383, Leu382, Phe130, Phe487, and Phe381. Thus, this compound can be considered as potential ACE inhibitor molecule. The docking complex and docking interactions of Compound-7 within the active site of ACE is shown in Fig. 3g.

\section{Docking interactions of Compound-8}

The Compound-8 showed the docking score of $-17.1659 \mathrm{~kJ} / \mathrm{mol}$ with the ACE. It is observed that the Compound-8 exhibited critical interactions with the catalytic amino acid residues present in the active site cavity of the ACE by favoring the Hbond interactions and non-bonded interactions. The amino acid Phe381, Leu380, and Arg110 favored the Hbond interactions and the non-bonded interactions are supported by Arg110, Glu383, Leu382, Phe130, Phe487, and Phe381. Thus, this compound can be considered as potential ACE inhibitor molecule. The docking complex and docking interactions of Compound-8 within the active site of ACE is shown in Fig. 3h.

\section{Docking interactions of Compound-9}

The Compound-9 showed the docking score of $-15.7295 \mathrm{~kJ} / \mathrm{mol}$ with the ACE. It is observed that the Compound- 9 exhibited critical interactions with the catalytic amino acid residues present in the active site cavity of the ACE by favoring the Hbond interactions and non-bonded interactions. The amino acid Phe381, Leu380, and Arg110 favored the Hbond interactions and the non-bonded interactions are supported by 


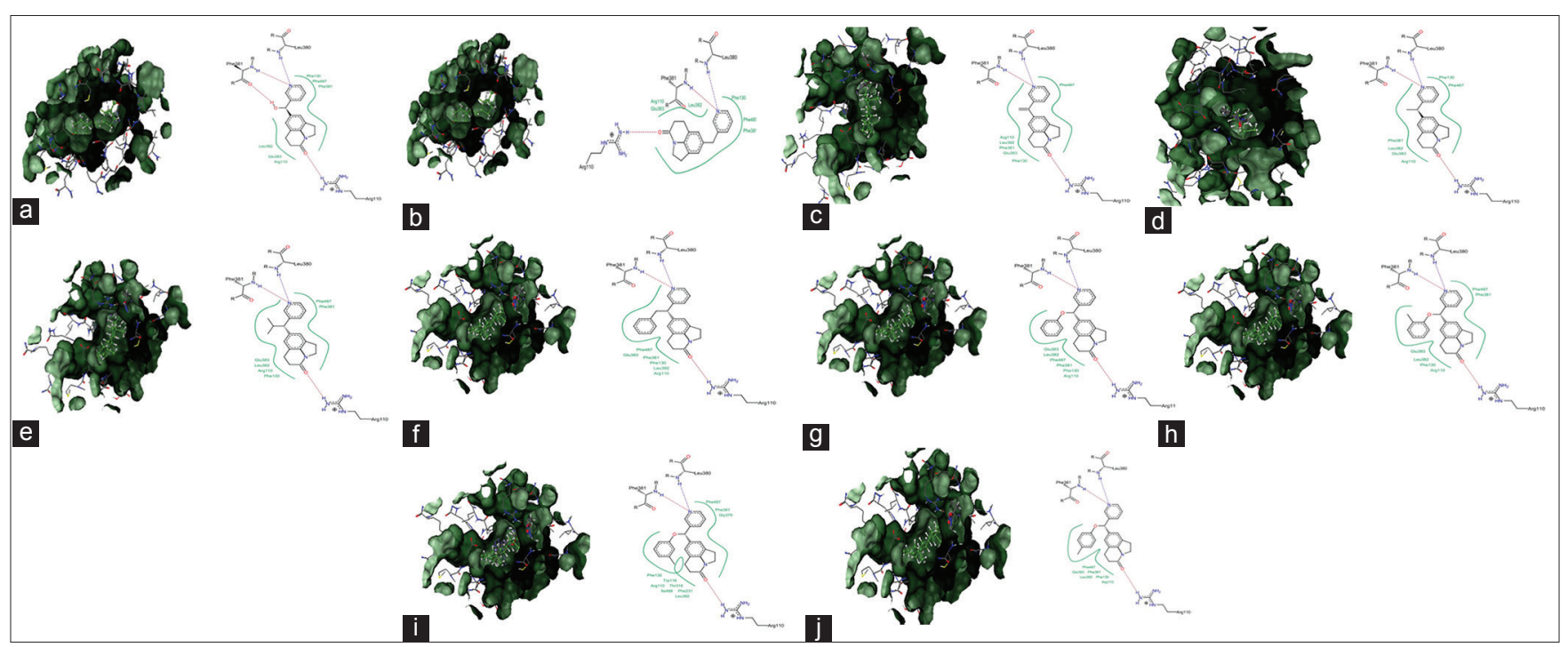

Fig. 3 (a) Docking complex and interactions of Compound-1 with angiotensin-converting enzyme (ACE) (Score: $-11.3060 \mathrm{~kJ} / \mathrm{mol}$ ). (b) Docking complex and interactions of Compound-2 with ACE (Score: $-\mathbf{1 7 . 9 4 6 9} \mathrm{kJ} / \mathrm{mol}$ ). (c) Docking complex and interactions of Compound-3 with ACE (Score: $\mathbf{- 1 8 . 9 1 0 0 ~ k J / m o l ) . ~ ( d ) ~ D o c k i n g ~ c o m p l e x ~ a n d ~ i n t e r a c t i o n s ~ o f ~ C o m p o u n d - 4 ~ w i t h ~ A C E ~}$

(Score: $-26.6290 \mathrm{~kJ} / \mathrm{mol}$ ). (e) Docking complex and interactions of Compound-5 with ACE (Score: $-14.2283 \mathrm{~kJ} / \mathrm{mol}$ ). (f) Docking complex and interactions of Compound- 6 with ACE (Score: $-11.1612 \mathrm{~kJ} / \mathrm{mol}$ ). (g) Docking complex and interactions of Compound-7 with ACE (Score: $-15.9906 \mathrm{~kJ} / \mathrm{mol}$ ). (h) Docking complex and interactions of Compound-8 with ACE (Score: $-17.1659 \mathrm{~kJ} / \mathrm{mol}$ ). (i) Docking complex and interactions of Compound-9 with ACE (Score: $-15.7295 \mathrm{~kJ} / \mathrm{mol}$ ). (j) Docking complex and interactions of Compound-10 with ACE

(Score: $\mathbf{- 1 4 . 7 7 6 1 ~ k J / m o l ) ~}$

Phe487, Phe381, Gly379, Phe130, Trp116, Thr318, Phe231, Leu382, Ile488, and Arg110. Thus, this compound can be considered as potential ACE inhibitor molecule. The docking complex and docking interactions of Compound-9 within the active site of ACE is shown in Fig. 3i.

\section{Docking interactions of Compound-10}

The Compound-10 showed the docking score of $-14.7761 \mathrm{~kJ} / \mathrm{mol}$ with the ACE. It is observed that the Compound-10 exhibited critical interactions with the catalytic amino acid residues present in the active site cavity of the ACE by favoring the Hbond interactions and non-bonded interactions. The amino acid Phe381, Leu380, and Arg110 favored the Hbond interactions and the non-bonded interactions are supported by Arg110, Glu383, Leu382, Phe130, Phe487, and Phe381. Thus, this compound can be considered as potential ACE inhibitor molecule. The docking complex and docking interactions of Compound-10 within the active site of ACE is shown in Fig. 3j.

\section{DFT studies}

Among the selected 10 ACE inhibitors, the Compound- 4 exhibited the highest docking score, and thus, the Compound-4 was considered to explore its structural features and also the chemical entities required for the activity of the compound. The compound geometry was analyzed through predicting Fourier transform infrared (FT-IR) and FT Raman spectra of the optimized Compound-4. Further, considering that the determination of the energy gap between highest occupied molecular orbital (HOMO) and lowest unoccupied molecular orbitals (LUMO) could provide the chemical reactivity and kinetic stability of molecules, the Compounds 4 was optimized and proposed for theoretical calculations (DFT studies) using Gaussian software.

\section{Vibrational analysis}

The vibration spectral analysis is performed on the basis of the characteristic vibrations of the amino group, hydroxyl group, carbonyl group, and methyl group. The optimized 3D structure of Compound-4 is shown in Fig. 4.

The theoretically predicted FT-IR and FT Raman spectra using B3LYP/6-311 G $(\mathrm{d}, \mathrm{p})$ method and the observed experimental FT-IR and FT Raman spectra are shown in Figs. 5 and 6, respectively. The

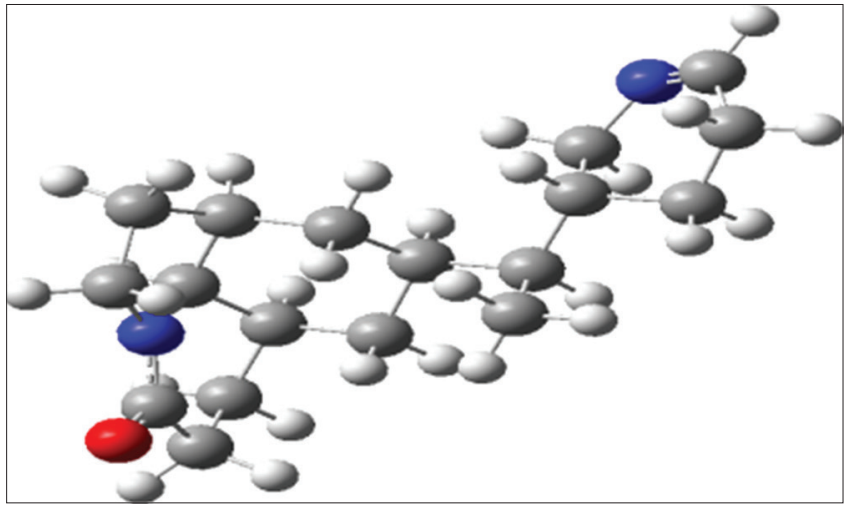

Fig. 4: The optimized geometry of the Compound-4

respective FT-IR spectra and FT Raman spectra were recorded in the range from 4000 to $400 \mathrm{~cm}^{-1}$.

The Compound-4 exhibits a sharp peak at $2000 \mathrm{~cm}^{-1}$ associated with the $-\mathrm{C}-\mathrm{H}-$ stretching vibrations of $\mathrm{CH}$ and $\mathrm{CH}_{3}$ groups. The signal characteristic bands of $\mathrm{C}=\mathrm{O}$ (carbonyl) stretching vibration at 1600 $1750 \mathrm{~cm}^{-1}$ are due to the presence of some aromatic esters.

The compounds containing methyl groups and $\mathrm{C}-\mathrm{H}$ groups are electron donating substituent in the aromatic ring system. Absorption arising from C-H stretching in the alkenes occurs in the region $3000-2840 \mathrm{~cm}^{-1}$. The lowering of the wave numbers from the normal values is due to the intermolecular charge transfer from the dimethyl group to the hydroxyl group through $\pi$ conjugation system.

The changes in intensity of the $\mathrm{CH}_{3}$ stretching mode are also due to the influence of electronic effects resulting from hyperconjugation of methyl group with nitrogen atom and the aromatic ring system. Thus, the hyperconjugation of methyl group, causing changes in intensity in IR, clearly indicates that methyl hydrogen is directly involved in the donation of electronic charge. The asymmetric and symmetric bending 
vibrations of methyl group normally appear around 1446 and $1375 \mathrm{~cm}^{-1}$, respectively. The relatively large value of intensity in IR and Raman wave numbers of the rocking modes suggests the presence of hyperconjugation. The twisting and torsion vibrations are identified below $503 \mathrm{~cm}^{-1}$, which are also supported by the computed wave numbers.

The fundamental modes involving the amino group are stretching and bending of $\mathrm{NH}$ bonds, torsion, and inversion. The Compound-4 under investigation possesses one $\mathrm{NH} 2$ group, and hence, one expects one symmetric and one asymmetric $\mathrm{N}-\mathrm{H}$ stretching vibrations. In all the primary aromatic amines, the $\mathrm{N}-\mathrm{H}$ stretching frequency occurs in the region $3300-3500 \mathrm{~cm}^{-1}$ and. The antisymmetric stretching mode appears to be higher wave number than the symmetric. In Compound-4, the $\mathrm{NH}_{2}$ asymmetric stretching vibration is observed in IR at 2500 as a medium band; the symmetric stretching vibration is observed in IR around 3520 $\mathrm{cm}^{-1}$ and in Raman around $3580 \mathrm{~cm}^{-1}$. The frequency lowering present in the molecule is due to the intermolecular interaction.

The ring modes which are found to be active in both IR and Raman spectra enable the molecule to be an effective $\pi$ conjugation system. Due to the existence of one directional charge transfer from the donor to the acceptor through the single-double bond conjugated path induces large variations of both the molecular dipole moment and the molecular polarizability during the same vibrations, thus gaining the activity of both IR and Raman at the same time. The simultaneous activation of IR and Raman ring modes also supports the charge transfer interaction between $\mathrm{CH}_{3}$ and the aromatic ring which also plays an important role in the biological activity of the compound to establish as a pharmaceutical compound with inhibitory activities.

\section{Natural bond analysis (HOMO-LUMO)}

The Eigenvalue of LUMO-HOMO energy gap reflects the chemical activity of the molecule. The HOMO-LUMO plot of Compound-4 is

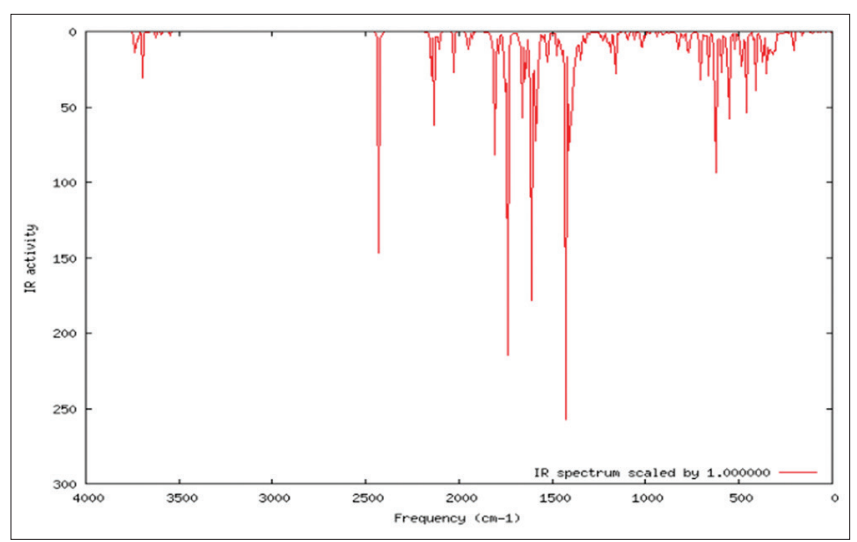

Fig. 5: Theoretical Fourier transform infrared of Compound-4

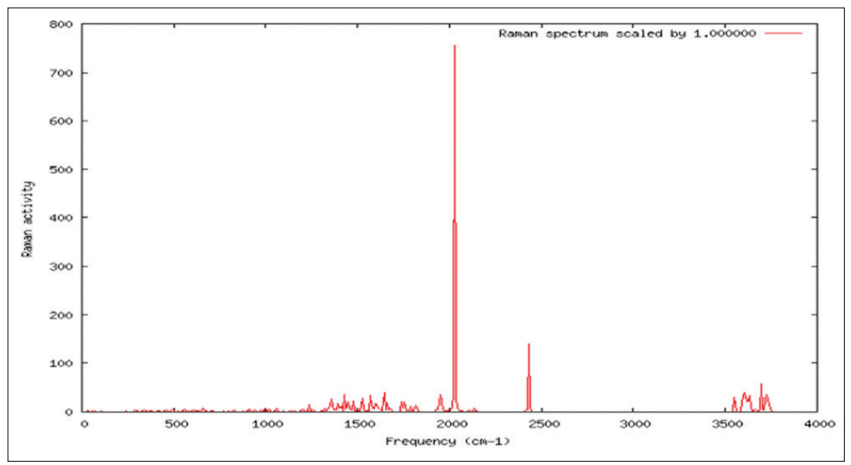

Fig. 6: Theoretical Fourier transform Raman spectra of Compound-4 shown in Fig. 7. The calculated HOMO of $-4.46 \mathrm{eV}$ and LUMO of -2.86 $\mathrm{eV}$ are observed.

The decrease in the HOMO and LUMO energy gap (1.60 eV) explains the eventual charge transfer interaction taking place within the molecule, due to the strong electron-accepting ability of the electron-acceptor group. It is worth noting that HOMOs have an overall $\pi$ bonding character along with a considerable non-bonding character and LUMOs have an antibonding $\pi^{*}$ character. The strong charge transfer interaction is responsible for the bioactivity of the molecule.

\section{Molecular electrostatic potentials}

The electrostatic contour map of the Compound-4 is shown in Fig. 8. The blue contours indicate electropositive charges correlating with activity and the Hbond donor regions, and the red contour indicates the relationship between negative charge and activity and also the Hbond acceptor regions.

The increase in positive charge and H-bond donor regions are favored in blue region while increase in negative charge and $\mathrm{H}$-bond acceptor regions are favored in red region. Blue-colored regions show areas where electropositive charged groups enhance inhibitory activity by the presence of $\mathrm{H}$-bond donors, while red regions represent where electronegative charged groups improve the activity with the presence of $\mathrm{H}$-bond acceptors. The green colored contours indicates the steric bulk groups that are required to increase the activity while the yellow contours indicates the unfavorable steric bulk groups that can decrease the activity.

Thus, considering the docking interactions of Compound-4 with ACE protein and geometry, HOMO-LUMO energy gaps and electrostatic contour map of the Compound-4 clearly envisage that the presence of electropositive charged groups is very essential to enhance the inhibitory activity along with the H-bond donors, and the presence of electronegative charged groups can possibly improve the activity along the presence of $\mathrm{H}$-bond acceptors.

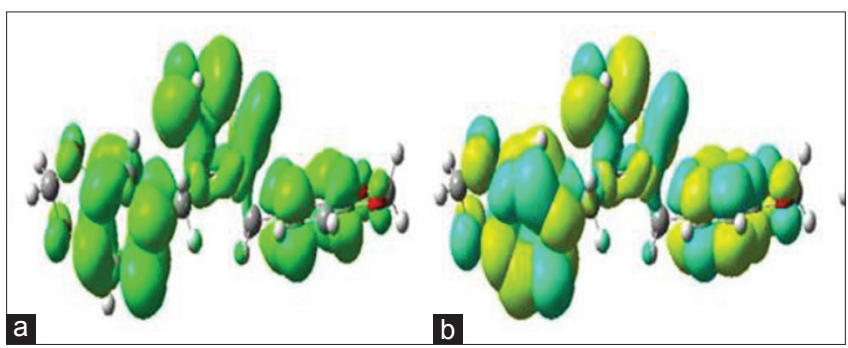

Fig. 7: The highest occupied molecular orbital-lowest unoccupied molecular orbitals plot of the Compound-4

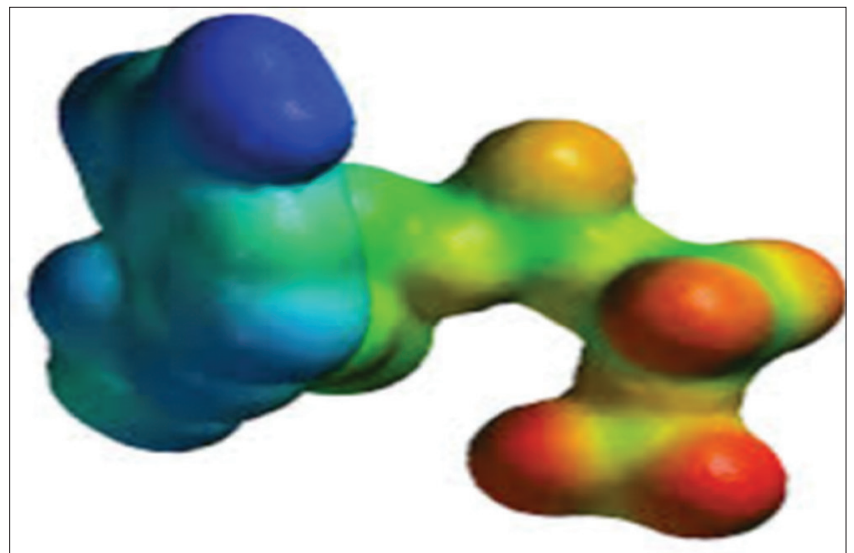

Fig. 8: Electrostatic contour map of the Compound-4 


\section{CONCLUSION}

Hypertension is an important risk factor, contributing to other cardiovascular diseases such as blood vessel disorders, coronary heart disease, aortic aneurysm, and stroke. The search for many novel ACE inhibitors is in progress that can be effective in the treatment of hypertension. Hence, the clear understanding of the present ACE inhibitors might give envisage the required chemical entities for the better ACE inhibition activity. Thus, in this study, a set of ACE inhibitors is used to explore their binding mechanisms with ACE protein and the Compound- 4 with the highest activity is considered for further DFT analysis. The DFT studies revealed the HOMO $(-4.46 \mathrm{eV})$ and LUMO $(-2.86 \mathrm{eV})$ and the energy gap $(1.60 \mathrm{eV})$. This NBO analysis explained the eventual charge transfer is due to the strong electron-accepting ability of the compound. Furthermore, the electrostatic contour map revealed that the presence of electropositive charged groups is very essential to enhance the inhibitory activity along with the H-bond donors, and the presence of electronegative charged groups can possibly improve the activity along the presence of $\mathrm{H}$-bond acceptors.

\section{AUTHORS' CONTRIBUTIONS}

CZDL carried out of the experimental part of the work. The CV and ASM guided and monitored the experimental design; GS carried the data compilation and drafted the manuscript.

\section{CONFLICTS OF INTEREST}

The authors do not have any conflicts of interest to declare.

\section{REFERENCES}

1. Clark RD, Strizhev A, Leonard JM, Blake JF, Matthew JB. Consensus scoring for ligand/protein interactions. J Mol Graph Model 2002;20:281-95.

2. Gehlhaar DK, Verkhivker G, Rejto PA, Fogel DB, Fogel LJ. Docking Conformationally Flexible Small Molecules into a Protein Binding Site through Evolutionary Programming. Proceedings of the Fourth International Conference on Evolutionary Programming; 1995. p. 615-27.

3. Charifson PS, Corkery JJ, Murcko MA, Walters WP. Consensus scoring: A method for obtaining improved hit rates from docking databases of three-dimensional structures into proteins. J Med Chem 1999; 42:5100-9.

4. Izzo JL, Black HR, Goodfriend TL. Hypertension Primer: The Essentials of High Blood Pressure. Philadelphia, PA: Lippincott Williams and Wilkins; 2003.
5. Advanced Chemistry Development, Inc. ACD/Chem Sketch Freeware. Version 12. Toronto, ON, Canada: Advanced Chemistry Development, Inc. Available from: http://www.acdlabs.com.

6. Weininger D. SMILES, a chemical language and information system. Introduction to methodology and encoding rules. J Chem Inf Comput Sci 1998;28:31-6.

7. Natesh R, Schwager SL, Sturrock ED, Acharya KR. Crystal structure of the human angiotensin-converting enzyme-lisinopril complex. Nature 2003;421:551-4.

8. Sussman JL, Lin D, Jiang J, Manning NO, Prilusky J, Ritter O, et al. Protein data bank (PDB): Database of three-dimensional structural information of biological macromolecules. Acta Crystallogr D Biol Crystallogr 1998;54:1078-84.

9. Rarey M, Kramer B, Lengauer T, Klebe G. A fast flexible docking method using an incremental construction algorithm. J Mol Biol 1996;261:470-89.

10. Gnanendra S, Anusuya S, Natarajan J. Molecular modeling and active site analysis of sdiA homolog, a putative quorum sensor for salmonella typhimurium pathogenecity reveals specific binding patterns of AHL transcriptional regulators. J Mol Model 2012;18:4709-19.

11. Sari BL, Mun'im A, Yanuar A, Riadhi R. Screening of $\alpha$-glucosidase inhibitors from Terminalia catappa L. fruits using molecular docking method and in vitro test. Int J Pharm Pharm Sci 2016;8:184-9.

12. Anjugam C, Sridevi M, Gnanendra TS. Structure-based docking studies toward exploring the potential anticancer activity of morin against nonmelanoma skin cancer therapeutic drug targets. Asian J Pharm Clin Res 2018;11:61-6.

13. Stierand K, Maass PC, Rarey M. Molecular complexes at a glance: Automated generation of two-dimensional complex diagrams. Bioinformatics 2006;22:1710-6.

14. Suresh DM, Amalanathan M, Sebastian S, Sajan D, Hubert Joe I, Bena Jothy $\mathrm{V}$, et al. Vibrational spectral investigation and natural bond orbital analysis of pharmaceutical compound 7-amino-2,4dimethylquinolinium formate-DFT approach. Spectrochim Acta A Mol Biomol Spectrosc 2013;115:595-602.

15. White WB, Prisant LM, Wright JT Jr. Management of patients with hypertension and diabetes mellitus: Advances in the evidence for intensive treatment. Am J Med 2000;108:238-45.

16. Vázquez-Valadez VH, Abrego VH, Martínez PA, Torres G, Zúñiga O, Escutia D, et al. Docking studies of methylthiomorpholin phenols (LQM300 series) with angiotensin-converting enzyme (ACE). Open Med Chem J 2013;7:30-8. 